\title{
Processing of TerraSAR-X/TanDEM-X \\ High Resolution Radar Data Using \\ Persistent Scatterers Method
}

\author{
Anton V. Filatov*a, Arkadi V. Yevtyushkin ${ }^{a}$, \\ Yuri V. Vasilev ${ }^{b}$ and Peter V. Pogodin \\ ${ }^{a}$ Immanuel Kant Baltic Federal University \\ 14 A. Nevskogo Str., Kaliningrad, 236016, Russia \\ ${ }^{b}$ West Siberian Division of Trofimuk Institute \\ of Petroleum-Gas Geology and Geophysics SB RAS \\ 56 Volodarskogo Str., Tyumen, 625000, Russia \\ 'Samotlorneftegaz \\ 4 Lenin Str., Nizhnevartovsk, 628606, Russia
}

Received 30.03.2015, received in revised form 07.02.2017, accepted 29.04.2017

Use of satellite multi-temporal radar measurements and method of persistent scatterers interferometry allow to achieve high accuracy of calculation of ground surface subsidence to the first millimeters. Given paper presents results of 13 TerraSAR-X/TanDEM-X radar images processing on territory of Samotlor oil field for the period from August to December, 2013. The comparison of results of processing with materials of ground geodetic measurements on points of the Samotlor geodynamic polygon is carried out.

Keywords: radar interferometry, persistent scatterers interferometry, geodynamic polygon, subsidence, TerraSAR-X, TanDEM-X.

Citation: Filatov A.V., Yevtyushkin A.V., Vasilev Yu.V., Pogodin P.V. Processing of TerraSAR-X/TanDEM-X high resolution radar data using persistent scatterers method, J. Sib. Fed. Univ. Eng. technol., 2018, 11(1), 86-94. DOI: 10.17516/1999-494X0012 .

(c) Siberian Federal University. All rights reserved

* Corresponding author E-mail address: anfilatov@kantiana.ru 


\title{
Обработка радарных данных высокого разрешения TerraSAR-X/TanDEM-X \\ по методу постоянных отражателей
}

\author{
А.В. Филатов ${ }^{\text {a }}$, А.В. Евтюшкин ${ }^{a}$, \\ Ю.В. Васильев ${ }^{\tilde{\sigma}}$, П.В. Погодин ${ }^{\mathrm{B}}$ \\ ${ }^{a}$ Балтийский федеральный университет им. И. Канта \\ Россия, 236016, Калининград, ул. А. Невского, 14 \\ бЗападно-Сибирский филиал Института нефтегазовой геологии \\ и геофизики им. А.А. Трофимука СО РАН \\ Россия, 625000, Тюмень, ул. Володарского, 56 \\ ${ }^{8} \mathrm{AO}$ «Самотлорнефтегаз» \\ Россия, 628606, Нижневартовск, ул. Ленина, 4
}

Использование спутниковых многовременных радарных измерений $u$ метода интерферометрии постоянных отражателей позволяет достичь высокой точности расчета смещений земной поверхности до первых миллиметров. В статье представлень результаты обработки 13 радиолокачионных кадров TerraSAR-X/TanDEM-X на территории Самотлорского нефтяного месторождения за период август-декабрь 2013 г. Проведено сравнение результатов обработки с результатами наземных геодезических измерений на пунктах Самотлорского геодинамического полигона.

Ключевые слова: радиолокационная интерферометрия, интерферометрия постоянных отражателей, геодинамический полигон, просадки, TerraSAR-X, TanDEM-X.

\section{Введение}

В странах ЕC, особенно в Италии, организован национальный мониторинг оползней и просадок на угольных шахтах по данным спутниковых радаров COSMO-SkyMed (CSM), TerraSAR-X (TSX), TanDEM-X (TDX) на основе технологии постоянных интерферометрических отражателей (PSI) [1-6]. В России только отдельные группы исследователей выполняют кратковременный мониторинг подвижек наиболее опасных техногенных территорий - Сочи, Березняки, Альметьевск, Кузбасс, Самотлор [7, 8]. Наведенная сейсмичность [9] в районах интенсивной угле- и нефтегазодобычи вызывает быстропротекающий процесс перестройки напряженного состояния верхних горизонтов дневной поверхности, для мониторинга которого целесообразно применять технологию PSI с минимальными периодами наблюдений.

Реализация метода интерферометрии устойчивых отражателей на кластере на основе StaMPS позволила превзойти по скорости обработки известные интерферометрические пакеты [10]. Предлагаемая технология интерферометрических расчетов на кластере позволяет организовать в одном вычислительном центре непрерывную обработку и мониторинг десятков площадей наблюдений по коротко-периодическим радарным данным, доступным по экономическим возможностям в РФ, - TSX, TDX, Sentinel-1A, Palsar-2.

В настоящей статье рассмотрены особенности интерпретации результатов обработки по технологии PSI высокопериодических радарных данных TSX/TDX и наземных геодезических измерений за 2013-2014 гг. при мониторинге мульды оседания Самотлорского месторождения. 


\section{Методы обработки}

Для мониторинга объектов нефтедобычи, расположенных на месторождениях, реализован метод интерферометрической обработки постоянных отражателей, описанный в работах [7, $10,11]$. Данный метод предусматривает алгоритмы выбора постоянных отражателей, расчета атмосферного фазового сдвига, скорости смещений и высот. Постоянным отражателем считается объект, который дает высокий и стабильный уровень обратного отражения (амплитуда пиксела), тогда фаза сигнала, принятого от такого объекта, имеет низкую дисперсию.

Рассмотрим составляющие интерферометрической фазы при обработке двух разновременных съемках (РЛИ).

$$
\begin{aligned}
& \phi_{k}(\vec{x})=\phi_{r k}(\vec{x})+\phi_{\mu k}(\vec{x})+\phi_{a k}(\vec{x})+\phi_{\sigma k}(\vec{x})= \\
& =\phi_{q k}(\vec{x})+\phi_{s k}(\vec{x})+\phi_{\mu k}(\vec{x})+\phi_{a k}(\vec{x})+\phi_{\sigma k}(\vec{x}),
\end{aligned}
$$

где каждая компонента представляется формулой:

$$
\begin{aligned}
& \phi_{r k}(\vec{x})=\frac{4 \pi}{\lambda} \Delta r_{k}(\vec{x}), \\
& \phi_{\mu k}(\vec{x})=\frac{4 \pi}{\lambda} \Delta \mu_{k}(\vec{x}), \\
& \phi_{a k}(\vec{x})=\frac{4 \pi}{\lambda}\left(a_{k}(\vec{x})-a_{m}(\vec{x})\right), \\
& \phi_{\sigma k}(\vec{x})=\frac{4 \pi}{\lambda}\left(\sigma_{k}(\vec{x})-\sigma_{m}(\vec{x})\right), \\
& \phi_{q k}(\vec{x})=\frac{4 \pi}{\lambda R} B_{k}(\vec{x}) \frac{q(\vec{x})}{\sin \alpha}, \\
& \phi_{s k}(\vec{x})=\frac{4 \pi}{\lambda R} B_{k}(\vec{x}) \frac{\eta}{\tan \alpha},
\end{aligned}
$$

где $\vec{x}=\left[\begin{array}{l}\xi \\ \eta\end{array}\right]$ - координаты пиксела ( $\xi$ - азимут, $\eta$ - наклонная дальность); $\phi_{r k}-$ фазовая компонента (ФК) за счет разности расстояний, проходимых сигналом при разновременной съемке; $\phi_{\mu k}-$ ФК за счет смещения объекта; $\phi_{a k}-$ ФК за счет различия атмосферных условий; $\phi_{\sigma k}-$ разница фазы переотражения; $\phi_{q k}-\Phi К$, зависящая от высоты объекта над опорной поверхностью; $\phi_{s k}-\Phi К$, зависящая от наклонной дальности объекта; $\Delta r_{k}$ - разность расстояний до объекта при разновременной съемке; $\Delta \mu_{k}$ - смещение объекта в направлении линии визирования за время между съемками; $a_{m}, a_{k}$ - изменение дистанции сигнала вследствие влияния атмосферы при исходной (master) и повторной (slave) съемках соответственно; $\sigma_{m}, \sigma_{k}-$ фаза переотражения master и slave соответственно; $\lambda$ - длина волны зондирующего сигнала; $R$ - расстояние между антенной радара и объектом при master-съемке; $B_{k}-$ перпендикулярная проекция базовой линии (расстояния между положениями радара); $q$ - высота; $\eta$ - наклонная дальность; $\alpha$ - угол обзора.

Для выбора на радиолокационных кадрах точек, соответствующих отражению сигнала от стабильных отражающих объектов, используется формула индекса дисперсии

$$
\sigma_{v} \approx \frac{\sigma_{A}}{m_{A}} \equiv D_{A},
$$


где $\sigma_{v}-$ дисперсия фазы; $\sigma_{A}-$ дисперсия амплитуды; $m_{A}-$ среднее значение амплитуды; $D_{A}-$ индекс дисперсии.

Для выбора постоянных отражателей устанавливается пороговое значение $D_{A}<0.4$.

\section{Используемые радиолокационные данные}

Преимущества метода постоянных отражателей перед применением площадной радарной интерферометрии основаны на особенных свойствах точечных объектов, которые сохраняют высокий уровень обратного отражения радиолокационного сигнала в течение множества последовательных съемок $[7,11]$. Зачастую размер такого отражающего объекта меньше элемента разрешения, поэтому когерентность достаточно высока $(>0.5)$ даже для пар кадров с базовой линией больше критической. При условии оценки и удаления атмосферного фазового сдвига высоты таких точек над опорной поверхностью могут быть рассчитаны с точностью лучше 1 м, а смещения - лучше 1 см.

Для мониторинга объектов нефтедобычи, расположенных на месторождениях, реализован метод интерферометрической обработки постоянных. Данный метод предусматривает алгоритмы выбора постоянных отражателей и расчета атмосферного фазового сдвига, скорости смещений и высот. Постоянным отражателем считается объект, который дает высокий и стабильный уровень обратного отражения (амплитуда пиксела), тогда фаза сигнала, принятого от такого объекта, имеет низкую дисперсию.

Для исследования смещений земной поверхности и техногенных объектов на территории Самотлорского лицензионного участка использованы спутниковые радиолокационные данные, полученные с двух спутников TSX, TDX, работающих в паре. Оператором спутников является Немецкий аэрокосмический центр (DLR).

Съемка территории Самотлорского месторождения радиолокаторами TSX/TDX проведена в период с августа по декабрь 2013 г. В обработке использованы 13 радиолокационных сцен размером 30 км в направлении наклонной дальности и 50 км в направлении азимута. Под покрытие попадают наиболее значимые районы, расположенные в площади горного отвода: центральная часть мульды оседания, г. Нижневартовск, Усть-Вахская площадь (Нижневартовская ГРЭС и пос. Излучинск).

\section{Результаты обработки}

Выбор основной сцены, относительно которой рассчитываются интерферограммы, основан на минимизации влияния факторов, снижающих когерентность интерферограммы: перпендикулярной базовой линии, временной базы и разницы доплеровских центроидов [10].

$$
\gamma_{m}=\frac{1}{K} \sum_{k=0}^{K} g\left(B_{k, m}, B_{c r}\right) \times g\left(T_{k, m}, T_{c r}\right) \times g\left(f d c_{k, m}, f d c_{c r}\right)
$$

где

$$
\begin{array}{r}
g(x, c)=\left\{\begin{array}{cc}
1-|x| / c & \text { при }|x|<c \\
0 & \text { при }|x| \geq c
\end{array}\right. \\
-89-
\end{array}
$$


$B_{k, m}, T_{k, m}, f d c_{k, m}$ - перпендикулярная пространственная база, временная база и разница доплеровских центроидов для каждой пары кадров; $B_{c r}, T_{c r}, f d c_{c r}$ - критические значения данных параметров.

Основной сценой выбрана съемка, произведенная 30 октября 2013 г.

В результате обработки 13 радиолокационных кадров TSX/TDX по методу интерферометрии постоянных отражателей определены скорости смещений (мм/год) и временные ряды смещений между последовательными съемками (мм). Расчет смещений проведен для 89290 точек, соответствующих техногенным объектам и элементам ландшафта земной поверхности. В районах городской и промышленной застройки смещения определены на основе плотной сети точек (от 200 точек/км²), для природных ландшафтов плотность устойчивых отражателей меньше. На рис. $1 a$ изображена карта скоростей смещений постоянных отражателей, полученная после применения дополнительной интерполяции точечных значений (метод локальных полиномов).

Интерполяция проведена таким образом, что в первую очередь учтены скорости смещений, расчитанных для плотных скоплений интерферометрических отражателей, что позволило значительно снизить ошибку, вносимую обособленными точками. Кроме того, интерполяция позволят получить сглаженную картину и построить контуры зон проседания и поднятия. При этом необходимо отметить, что скорость смещений каждого стабильного интерферометрического отражателя, отсносительно которых проводятся расчеты, - это линейная составляющая смещений точки в течение интервала наблюдений. На рис. $1 a$ цветовая шкала скоростей смещений представлена в мм/год, так как такое отображение дает возможнлость проводить анализ

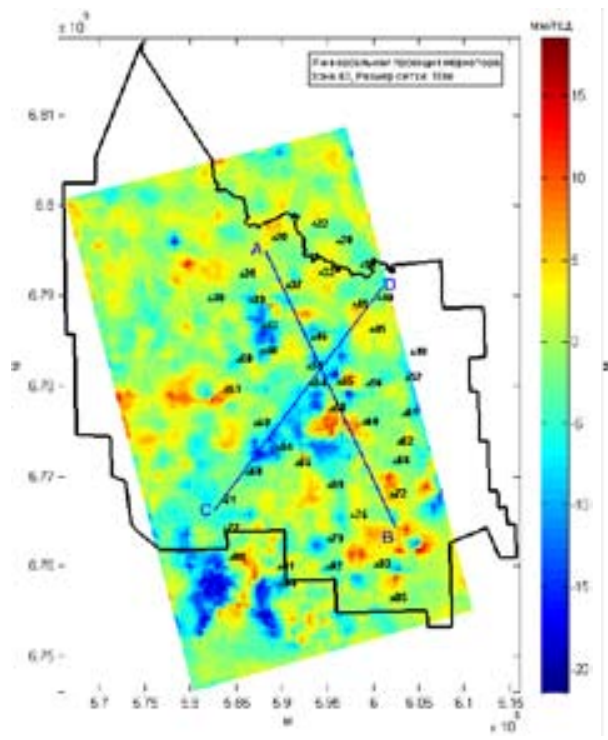

a

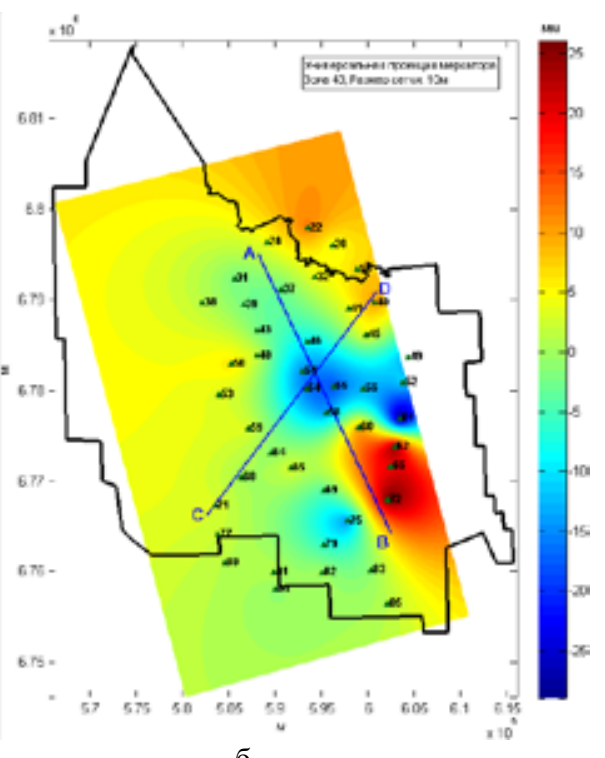

6

Рис. 1. Скорости смещений по результатам спутникового мониторинга (а) и смещения по данным наземных измерений (б) с профилями через мульду оседания

Fig. 1. Displacements velocity based on results of satellite monitoring (a) and ground measurements (b) with the profiles over the subsidence through 
и сравнение результатов космического мониторинга и наземных геодезических измерений за разные годы.

На карте скоростей смещений на рис. $1 a$ выделяются центральная часть мульды оседания Самотлорского месторождения и просадка на территории г. Нижневартовска. Зона поднятия южнее пункта 58 соответствует нескольким кустовым площадка в центре оз. Самотлор. Смещение пункта 58 по нивелировке -14 мм в 2012-2013 гг., самый ближайший к пункту отражающий объект за время спутникового мониторинга (4 месяца) сместился на -7 мм. Но несмотря на это южнее существует зона поднятия.

В ходе анализа детально рассмотрены две зоны. Первая соответствует Усть-Вахской площади, включая Нижневартовскую ГРЭС и пос. Излучинск. На территории Нижневартовской ГРЭС обнаружены две зоны разнонаправленных смещений, восточная часть имеет положительное смещение, западная - отрицательное. Такие же результаты были получены в ходе предыдущего цикла обработки в 2012 г. на основе 18 радиолокационных кадров ALOS $\backslash$ PALSAR за 2007-2011 гг. с использованием метода обнаружения зон геодинамического риска $[10,12]$.

\section{Оценка точности результатов}

Метод интерферометрической обработки многовременных радиолокационных измерений для точечных отражающих объектов использует статистическую обработку амплитуднофазовых компонент сигнала, отраженного от одних и тех же объектов. За счет такой обработки теоретическая точности метода может достигать 1 мм при достаточном объеме съемок. В ходе расчетов дополнительно определяется точность восстановление смещений отражающих объектов. Значение точности зависит от соотношения сигнал-шум для каждого отражающего объекта, а также от точности оценки и устранения таких компонент интерферометрической фазы, как атмосферный сдвиг, рельеф, ошибка определения орбиты. В ходе обработки 13 сцен TSX/ TDX достигнута точность определения скоростей смещений 2,5 мм/год.

Дополнительно проведено сравнение скоростей смещений, представленных на рис. $1 a$ с результатами наземных геодезических измерений на пунктах Самотлорского геодинамического полигона (смещения по нивелировке 2012-2013 гг.). Для определения точности выбран вариант, при котором в качестве смещений по нивелировке используется разница высот пунктов между 2012 и 2013 гг., а смещения по спутниковому мониторингу экстраполируются до годовых значений на основе результатов наблюдений за 4 месяца. Среднеквадратичное отклонение значений, рассчитанных по радиолокационным данным, от смещений пунктов полигона, измеренных по нивелировке, для 43 точек составило 11 мм/год.

На рис. 16 для сравнения с картой смещений по интерферометрии (рис. $1 a$ ) приведен результат интерполяции смещений пунктов Самотлорского геодинамического полигона (по нивелировке 2012-2013 гг.). Треугольниками с номерами отмечено положение 43 пунктов полигона, для которых проводилось сравнение со смещениями по интерферометрии. Линии $\mathrm{AB}$ и $\mathrm{CD}$ отмечают расположение пространственных профилей через мульду оседания. Смещения вдоль линий $\mathrm{AB}$ и $\mathrm{CD}$ изображены на рис. 2.

На рис. 3 представлены смещения отражающих объектов на территории г. Нижневартовска за время спутниковых наблюдений. В качестве растровой основы использован оптический снимок спутника QuickBird за 20 мая 2005 г. 

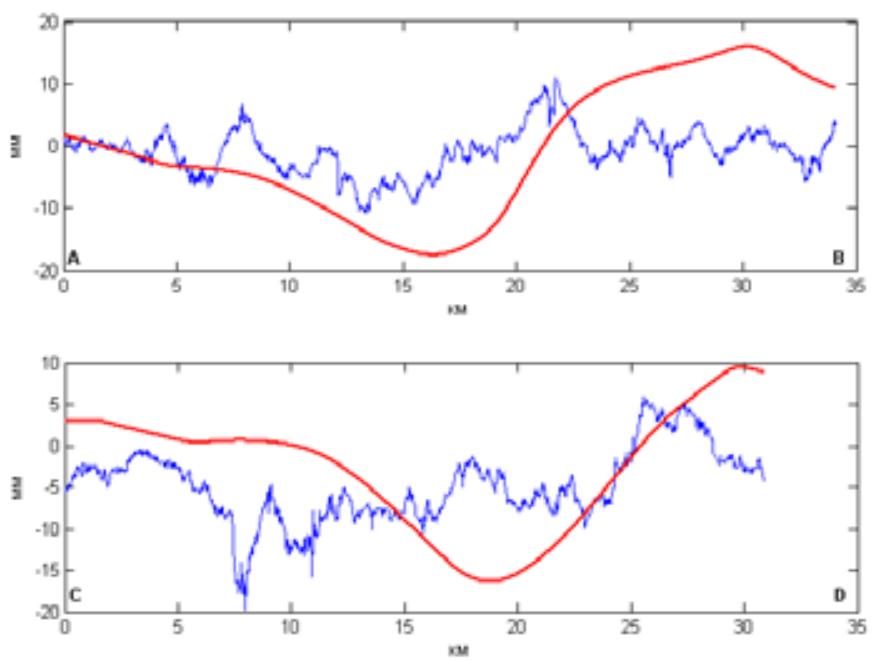

Рис. 2. Смещения по профилям через мульду оседания на основе геодезических измерений (красная линия) и радарного мониторинга (синяя линия)

Fig. 2. Displacements along the profile over the subsidence through based on geodetic measurements (red line) and radar observations (blue line)

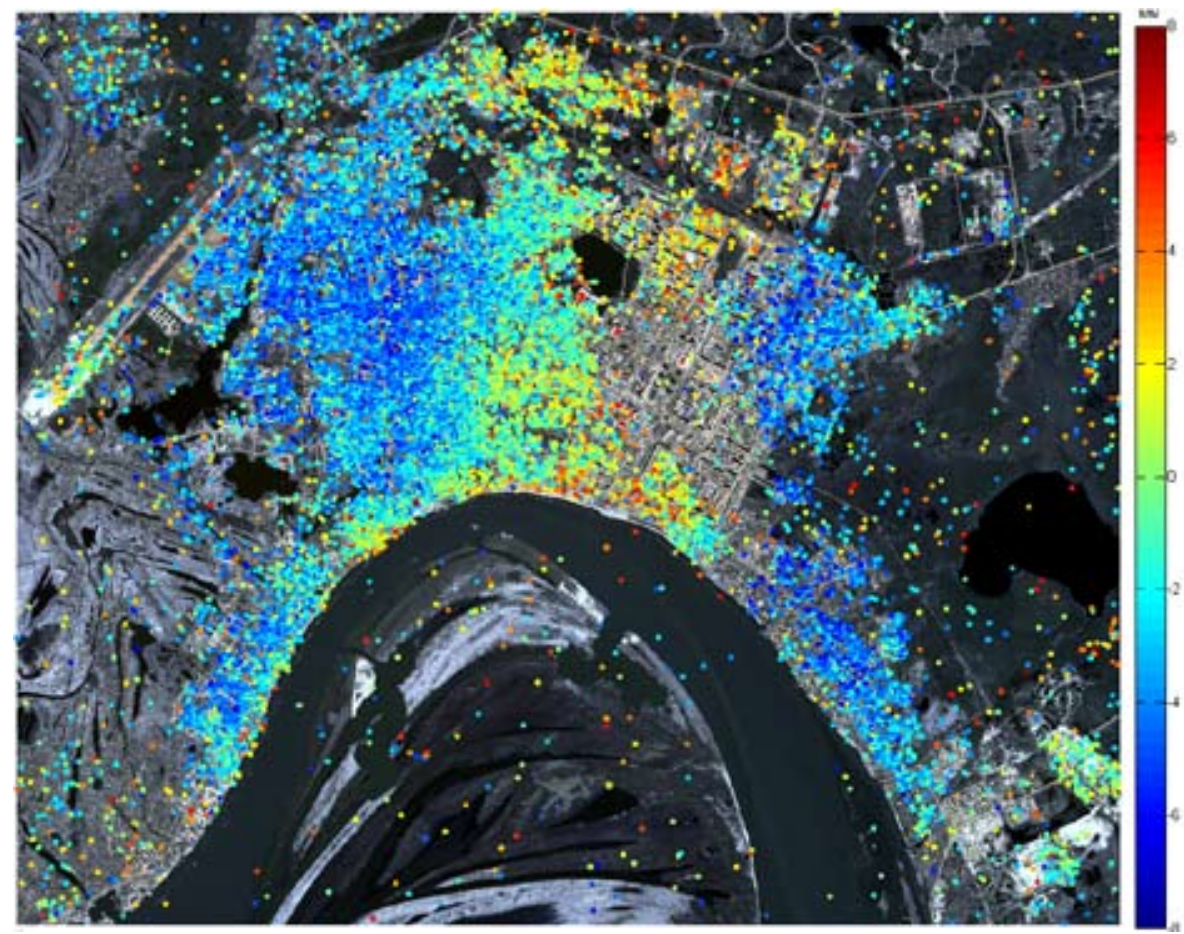

Рис. 3. Смещения стабильных интерферометрических отражателей на территории г. Нижневартовска за август-декабрь 2013 г. Растровая основа QuickBird 20.05.2005

Fig. 3. Displacements of persistent scatterers in the city of Nizhnevartovsk for the period from August to December 2013. The raster background is the QiuckBird image 20.05.2005 


\section{Заключение}

По результатам интерферометрической обработки 13 радиолокационных сцен спутников TSX/TDX, снятых в период с августа по декабрь 2013 г. с интервалом в 11 сут. на территории Самотлорского месторождения, сделаны следующие выводы:

1. Метод позволяет расчитывать смещения стабильных отражающих объектов в проекции на направление обзора радиолокатора на основе данных многопроходной съемки одной и той же территории.

2. Смещения рассчитаны для более плотной сети точек по сравнению с плотностью расположения пунктов геодинамического полигона. Большая плотность ( 200 точек/км²) достигнута в районах гражданской и промышленной застройки, а также в местах расположениях крупных объектов нефтедобычи.

3. Результаты спутникового мониторинга позволяют уточнить границы мульды оседания на территории, не охваченной наземными геодезическими измерениями.

4. Точность расчета скоростей смещений стабильных отражающих объектов, определенная на основе соотношения сигнал/шум для многовременных радиолокационных измерений, составила 2,5 мм/год.

5. Среднеквадратическая ошибка скоростей смещений, определенная с использованием данных по нивелировке за 2012-2013 гг. на Самотлорском геодинамическом полигоне в качестве эталонных, составила 11 мм/год.

6. Обнаружена корреляция между материалами наземных геодезических измерений на пунктах Самотлорского геодинамического полигона и результатами интерферометрической обработки спутниковых данных.

7. Обнаружена корреляция между смещениями, определенными по результатам обработки 18 кадров ALOS PALSAR за 2007-2011 гг. (предыдущих цикл горно-экологического мониторинга) и по текущим результатам по всей области и, в частности, для территории Нижневартовской ГРЭС. Восточная часть имеет среднее смещение +5 мм, западная -5 мм за 4 месяца съемки TSX/TDX.

8. Вследствие того, что определяет смещения отражающих объектов, на значения смещений оказывает влияние специфика самих объектов (температурное расширение/сжатие, диэлектрический свойства самой отражающей поверхности). Смещение совокупности близкорасположенных объектов является отражением подвижек земной поверхности.

\section{Работа выполнена при поддержке гранта РФФИ 16-37-00224 и проекта DLR GEO1978.}

\section{Список литературы}

[1] Prati C., Ferretti A., Perissin D. Recent advances on surface ground deformation measurement by means of repeated space-borne SAR observations. Journal of Geodynamics, 2010, 49, 161-170.

[2] Lagios E., Sakkas V., Novali F., Bellotti F., Ferretti A., Vlachou K., Dietrich V. SqueeSARTM and GPS Ground Deformation Monitoring of Santorini Volcano (1992-2012). Tectonophysics, 2013, $594,38-59$. 
[3] Bovenga F., Wasowski J., Nitti D.O., Nutricato R., Chiaradia M.T. Using Cosmo/SkyMed X-band and ENVISAT C-band SAR interferometry for landslide analysis. Remote Sensing Environment, 2012, 119, 272-285.

[4] Canuti P., Casagli N., Ermini L., Fanti R., Farina P. Landslide activity as a geoindicator in Italy: significance and new perspectives from remote sensing. Environmental Geology, 2004, 45, 907-919.

[5] Michalis P., Giourou A., Charalampopoulou B., Li Zh., Li Y. Land subsidence in southwest Cyprus revealed from C-band radar interferometry. Proceedings of SPIE 9229, Second International Conference on Remote Sensing and Geoinformation of the Environment (RSCy2014), 2004.

[6] Graniczny M., Colombo D., Kowalski Z., Przyłucka M., Zdanowski A. A. New results on ground deformation in the Upper Silesian Coal Basin (Southern Poland) obtained during the DORIS Project (EU-FP 7). Pure and Applied Geophysics, 2014.

[7] Bryksin V.M., Filatov A.V., Yevtyushkin A.V. Using of SAR data and DInSar-PSInSar technique for monitoring Western Siberia and Arctic. Journal of Radio Electronics, 2012, 6, 1-53.

[8] Эпов М.И., Миронов В.Л., Чимитдоржиев Т.Н., Захаров А.И., Захарова Л.Н., Селезнев В.С., Еманов А.Ф., Еманов А.А., Фатеев А.В. Наблюдение просадок поверхности земли в районе подземных угольных выработок Кузбасса по данным радиолокационной интерферометрии ALOS PALSAR. Исследование Земли из космоса, 2012, 4, 26 [Ероv M.I., Mironov V.L., Chimitdorzhiev T.N., Zakharov A.I., Zakharova L.N., Seleznev V.S., Emanov A.A., Fateev A.V. Observations of ground surface subsidence in area of Kuzbass underground mines based on ALOS PALSAR radar interferometry data. Earth Observations from Space, 2012, 4, 26 (in Russian)].

[9] Еманов А.Ф., Еманов А.А., Фатеев А.В., Лескова Е.В., Шевкунова Е.В., Подкорытова В.Г. Крупнейший техногенный сейсмический процесс на Земле. Бачатское землетрясение 18.06.2013 г. (ML=6.1, Кузбасс). Интерэкспо Гео-Сибирь, 2014, 2, 80-84 [Emanov A.F., Emanov A.A., Fateev A.V., Leskova E.V., Shevkunova E.V., Podkorytova V.G. The largest technogenic seismic process on Earth. Bachatskoe earthquake 18.06.2013 ( $\mathrm{ML}=6.1$, Kuzbass). Interexpo GeoSiberia, 2014, 2, 80-84 (in Russian)].

[10] Филатов А.В., Евтюшкин А.В., Брыксин В.М., Васильев Ю.В., Юрьев М.Л., Белоносов А.Ю. Использование метода интерферометрии устойчивых отражателей при геодинамическом мониторинге Самотлорского месторождения. Маркщейдерский вестник, 2012, 4, 57-62 [Filatov A.V., Evtyushkin A.V., Bryksin V.M., Vasilev Yu.V., Yurev M.L., Belonosov A.Yu. The use of persistent scatterers interferometry technique for Samotlor oilfield geodynamic monitoring. Mine Surveying Bulletin, 2012, 4, 57-62 (in Russian)].

[11] Ferretti A., Prati C., Rocca F. Permanent scatterers in SAR interferometry. IEEE Transacations on Geoscience and Remote Sensing, 2001, 39, 1, 8-20.

[12] Пат. 2506606 Российская Федерация МПК: G01S5/02. Способ обнаружения зон геодинамического риска на основе данных радиолокационного зондирования земной поверхности. Филатов А.В., Евтюшкин А.В., Брыксин В.М., заявитель и патентообладатель Балтийский федер. ун-т им. И. Канта. № 2012135096/28; рег. 16.08.2012 [Pat. 2506606 The Russian Federation MPK: G01S5/02. Method of discovering geodynamical risk zones based on data of radiolocation sounding of the earth surface. Filatov A.V., Evtuyshkin A.V., Bryksin V.M., Immanuel Kant Baltic Federal University. 2012135096/28; reg. 16.08.2012 (In Russian)]. 